\title{
Articles
}

\author{
MARIA MEDINA-VICENT ${ }^{1}$
}

\section{Cuerpos y mercado en la era de la precariedad}

\author{
Bodies and Market in the Era of Precariousness
}

\section{RESUMEN}

La relación actual entre los cuerpos y el mercado se vuelve un tema de central importancia en el marco neoliberal, ya que atañe de forma directa a los derechos y las oportunidades vitales de los individuos. En este contexto de acuciada precariedad estamos asistiendo a una progresiva mercantilización de los cuerpos, especialmente los de las mujeres, que pasan a estar sujetos a las leyes de la oferta y la demanda. Además, dicho proceso no se produce solamente en una dimensión corporal, sino también referida a la conformación de las subjetividades contemporáneas. Así pues, es en el seno de un sistema como el neoliberal donde adquieren sentido ciertas prácticas como la gestación subrogada o las técnicas de reproducción asistida, sobre todo, porque es la propia lógica neoliberal la que alimenta su funcionamiento. En el presente artículo tratamos de reflexionar alrededor de una cuestión que consideramos central para dicha mercantilización de los cuerpos de las mujeres: su sentido en un contexto neoliberal de precariedad y individualismo.

Palabras clave: cuerpos, mujeres, mercantilización, neoliberalismo, precariedad.

\begin{abstract}
The current relationship between bodies and market becomes a central issue in the neoliberal framework, since it directly concerns the rights and vital opportunities of individuals. In this context of acute precariousness we are witnessing a progressive commodification of bodies, especially those of women, who become subject to the laws of supply and demand. In addition, this process does not occur only in a corporeal dimension, but also refers to the conformation of contemporary subjectivities. Thus, it is within a system such as the neo-liberal where certain practices such as surrogate pregnancy or assisted reproduction techniques acquire meaning, especially because it is the neoliberal logic itself that feeds its functioning. In this article we try to reflect on an issue that we consider central to the commodification of women's bodies: their meaning in a neoliberal context of precariousness and individualism.
\end{abstract}

Keywords: Bodies, women, commodification, neoliberalism, precariousness.

\section{Sumario}

- Introducción. 1.- La era de la precariedad. 2.- El sentido de la elección individual en los tiempos postfeministas. 3.- Cuerpos y dominación en el marco neoliberal. 4.- La cuestión de género desde una perspectiva multidisciplinar. - Conclusión. - Referencias bibliográficas.

1 Departamento de Filosofía y Sociología; Instituto Universitario de Estudios Feministas y de Género Purificación Escribano (Universitat Jaume I); medinam@uji.es. Maria Medina-Vicent se encuentra dentro del Proyecto de Investigación Científica y Desarrollo Tecnológico FFI2016-76753-C2-2-P, financiado por el Ministerio Español de Economía y Competitividad.; y del Proyecto de Investigación Científica y Desarrollo Tecnológico del Plan de Promoción de la Investigación de la Universitat Jaume I para el año 2016 UJI-A2016-04. 


\section{Introducción}

El cuerpo ha sido uno de los temas de reflexión más importantes de la teoría feminista en los siglos XX y XXI. Hoy, la mercantilización de los cuerpos femeninos parece estar a la orden del día. Esta realidad nos invita a reflexionar sobre ciertas cuestiones de gran relevancia social como por ejemplo las técnicas de reproducción asistida (Martí Gual, 2011, 2014), los vientres de alquiler (Nuño, 2016; Guerra Palmero, 2017), la prostitución (Nuño, De Miguel y Fernández, 2017), etc. y sus implicaciones para la lucha por la igualdad de género protagonizada por el feminismo. Todas estas prácticas impregnan los cuerpos de las mujeres de significados, valores y dinámicas sociales que reproducen una situación de desigualdad patente, ya que en gran medida dichos valores responden a la propia lógica neoliberal.

Así pues, resulta necesario enmarcar estas prácticas y procesos en un marco de sentido mayor referido a la sociedad neoliberal y los valores asociados al nuevo modelo de sujeto individualista y completamente despolitizado (Laval y Dardot, 2013; García Ferrer, 2017). Este contexto general nos muestra una realidad caracterizada por el riesgo, la incertidumbre y la precariedad. Además, si tomamos como centro de nuestra reflexión la situación de las mujeres en este marco, veremos que dicha lógica neoliberal acaba por afectar de forma transcendental a la mercantilización de los cuerpos de las mujeres. Aquí tiene mucho que decir la lógica de la elección individual y el logro meritocrático, que tal y como afirma la autora Ana de Miguel (2015), puede acabar por ocultar los mecanismos estructurales e ideológicos que condicionan las elecciones de las personas según su sexo y las posibilidades de establecer un proyecto vital factible. En relación, será importante aproximarnos a las implicaciones de los discursos postfeministas y tratar de abordar cómo se relaciona el feminismo con la lógica neoliberal. De este modo, podemos comprender de una forma más consciente las diversas problemáticas que derivan de dicha situación.

Atendiendo a las cuestiones que acabamos de plantear, el presente número de la revista Asparkía. Investigació Feminista titulado «Cuerpos en venta», aglutina reflexiones que examinan desde diferentes ámbitos -filosofía, sociología, historia, literatura, comunicación, etc.- el estudio crítico de la corporeidad femenina y los retos que suponen para la lucha feminista su actual mercantilización. Os invitamos encarecidamente a consultar las propuestas que conforman este monográfico, donde se tratan cuestiones centrales como la gestación subrogada, la prostitución o la congelación de óvulos.

\section{La era de la precariedad}

A la hora de comprender el marco de sentido en el que se produce la mercantilización de los cuerpos, sobre todo los de las mujeres, cabe prestar atención a la era de la precariedad, un tiempo en que la incertidumbre y el aumento del riesgo vital, junto al descenso en los derechos sociales, ha convertido la vida de las personas en trayectos de difícil tránsito. Esta realidad, en gran medida, tiende a ser vivida 
en clave de autoexigencia y responsabilización de uno/a mismo/a. Es decir, los sujetos se culpabilizan a ellos mismos por la imposibilidad de trazar un proyecto de vida factible. En este sentido, Muñoz y Santos (2018: 9) nos advierten de que se está produciendo «la absolutización de la precariedad vital como norma social para la mayoría de la población».

La precariedad pasaría así de ser una cuestión cíclica dentro del desarrollo global del sistema capitalista, a convertirse en la regla general sobre la que éste se sostiene (Carbajo, 2014). De este modo, la estabilidad de la etapa fordista sería más bien una anomalía histórica frente a la flexibilidad y la inseguridad de la época postfordista (Alonso y Fernández Rodríguez, 2018: 46), donde la precariedad se convierte en centro del sentido social compartido. Se trata de un escenario donde la precariedad no solamente va a afectar a las relaciones de trabajo, sino que va a transformar la noción de ciudadanía, de individuo, las dinámicas empresariales y estatales, así como la forma en que concebimos nuestro lugar en el mundo. De este modo, a través del «nuevo espíritu del capitalismo» estudiado por Luc Boltanski y Éve Chiapello (2002), se entiende que esta nueva etapa requiere de un compromiso con el sistema capitalista elaborado autónomamente por parte de los sujetos. Es decir, ahora la sujeción no se produce tanto por la represión como por la vivencia autónoma de los individuos. Se configuran nuevas subjetividades y relaciones de los sujetos con las instituciones sociales y económicas basadas en la autoexplotación.

La extensión del riesgo es uno de los principios centrales sobre los que se sostiene esta sociedad neoliberal y precaria. Del riesgo se deriva una incertidumbre constante que se traduce en la inseguridad del individuo a la hora de poder mantener un trabajo estable -entre otras muchas cuestiones- y una vida más o menos organizada. Esto refuerza el afán de competitividad, además del hecho de que la gestión del riesgo que antes corría a cargo del Estado y más tarde de las empresas, ahora corre a cargo del individuo. Alonso y Fernández Rodríguez (2013, 130-132) consideran que dicha precariedad actúa como un fenómeno disciplinario, una técnica biopolítica en términos foucaultianos, por la cual los/as trabajadores/as se encuentran abandonados a las exigencias del mercado capitalista. En cierto modo, la lógica argumental del precariado promueve la autoexplotación poniendo en el propio sujeto el miedo y la incertidumbre (Berardi, 2003), impidiéndole disponer libremente de su tiempo para diseñar un plan de vida factible.

Por tanto, los nuevos recorridos vitales que se emprenden en la etapa neoliberal comportan la subjetivación individual de la flexibilidad y la eventualidad, lógicas que afectan a la alimentación, la planificación del tiempo, las posibilidades de establecer una familia, la residencia y, en definitiva, la construcción de una realidad empresarial donde la gestión de las emociones de los/as empleados/as se vuelve central. Así, la progresiva desprotección de los individuos por parte de instituciones y la obligación de tomar decisiones forzadas que tienen pocas posibilidades de éxito, actúan aquí como factores de riesgo (Muñoz y Santos, 2017). De esta manera, se idealiza la predisposición al cambio del individuo, pero no se tienen en cuenta las condiciones materiales sobre las que este cambio -sujeto en el elecciones supuestamente libres y conscientes- se va a llevar a cabo, ni los costes emocionales 
y sociales que el fracaso tiene para estas personas. Cabe señalar que todas estas cuestiones tienen su traslación a la experiencia concreta del sujeto heterogéneo «mujeres», un ejemplo pueden ser el aumento de ciertas prácticas como la gestación subrogada o la congelación de óvulos, que permiten a las mujeres amoldarse de una forma más clara a las contradictorias exigencias del neoliberalismo mediante el control mercantil de sus cuerpos (Rottenberg, 2014).

En resumen, resulta importante establecer esta primera reflexión sobre el contexto neoliberal y la lógica que subyace a los nuevos modelos de sujetos y subjetividades, ya que es este contexto el que en cierto modo incentiva y promueve la mercantilización de los cuerpos, una cuestión en gran medida centrada en la lógica de la elección individual. Si focalizamos nuestra reflexión en el caso de las mujeres, esta realidad adquiere ciertos tintes distintivos, que suponen nuevos retos para la lucha feminista actual (Fraser, 1989, 1997, 2008, 2015; Reverter-Bañón, 2001, 2017). A continuación, trataremos de trazar algunas reflexiones alrededor de la intersección entre sociedad neoliberal y la posición de las mujeres en su seno; así como de la ambivalente relación que se da entre feminismos y neoliberalismo.

\section{El sentido de la elección individual en los tiempos postfeministas}

A partir de las cuestiones planteadas en el apartado anterior, podemos entrever que la situación vital de los sujetos en este marco neoliberal, en especial de las mujeres, resulta de gran riesgo. Sobre todo, en lo que se refiere a la verdadera libertad que subyace al proceso de toma de decisiones vitales. Es decir, ¿cómo de libres y conscientes pueden llegar a ser las decisiones que se toman en un contexto de gran precariedad? Ésta es una pregunta de difícil respuesta, y en gran medida, cada una de las aportaciones que se presentan en el presente monográfico titulado «Cuerpos en venta», tratan de empujar al público lector al planteamiento y la reflexión en torno a esta cuestión.

Así pues, nos podemos preguntar por los riesgos que derivan de la conformación de los sujetos femeninos como sujetos neoliberales y despolitizados en un marco postfeminista. Entendemos el término "postfeminismo» como una sensibilidad (Gill, 2007), cuyo rasgo central es el modo en que selectivamente los sujetos definen el feminismo como asumido y repudiado a la vez (McRobbie, 2004, 2009), es decir, la invocación del feminismo por parte de los sujetos sirve para hacer y deshacer dicho movimiento. Dentro de esta cuestión resulta de gran relevancia el proceso de resignificación que se lleva a cabo de la elección individual como sinónimo de lucha colectiva (Medina-Vicent, 2018). La lógica de la elección individual, también conocida como Choice Feminism (Hirshman, 2006) -que se enmarca en un marco de sentido mayor referido al feminismo neoliberal (Rottenberg, 2013)-, se refiere a los procesos individuales de toma de decisiones que llevan a cabo los sujetos y que hacen que el peso de la desigualdad de género se sitúe en la decisión individual de cada mujer.

Se trata de una forma popular del feminismo contemporáneo, que empodera a las mujeres a ver las elecciones que hacen siempre como políticamente 
aceptables, sean las que sean (Thwaites, 2017) Sin embargo, teniendo en cuenta las dimensiones tratadas en el apartado anterior, cabe poner entre interrogantes el discurso de la elección tan prolífico en estos tiempos, ya que puede contener una lógica neoliberal subyacente que reproduce las desigualdades de género y las injusticias a nivel global. Y es que, en gran medida, la posición desde la que se elabora y lanza el discurso de la elección, a pesar de parecer aglutinar al grueso de mujeres entendidas como un conjunto de individuales, nos remite a una visión privilegiada de los problemas sociales. Es decir, aquellas personas que tienen la capacidad de escoger con «verdadera» libertad cómo organizar su vida, pertenecen a un grupo privilegiado con unos condicionantes concretos que les permiten poder escoger si tener familia o no, si estudiar o no, o incluso poder votar o no. Sin embargo, debemos tener en cuenta que las experiencias de las mujeres cambian según se ven atravesadas por componentes de raza, clase social e incluso país de residencia. Por tanto, resulta contraproducente y poco realista considerar que el constructo «mujeres» está claramente definido y tiene un sentido unívoco, realizar esta operación supone perder de vista la heterogeneidad y complejidad de dicho movimiento. Además, cabe recordar que las posibilidades de cada cual de establecer un programa de decisiones totalmente libres y consciente se ven influenciadas por la influencia de la lógica de mercado sobre su vida. Una lógica que unos grupos humanos padecen de formas más violenta que otros.

A través de este tipo de feminismo, que se presenta como un feminismo tolerante con todas las decisiones que las mujeres tomen desde su aparente libertad, la discusión política se encuentra fuera de la ecuación (Ferguson, 2010). Y es que, en el objetivo por crear una sociedad más justa, no todas las decisiones pueden ser aceptadas si éstas reproducen las estructuras desiguales (Swirsky y Angelone, 2016), el compromiso de transformación social y política debe ser siempre la base de toda actuación que se considere feminista.

De este modo, esta nueva formulación del feminismo sirve al neoliberalismo en la medida en que sustituye la consecución de la justicia social por la consecución de la felicidad personal. Se despolitiza al movimiento, desarticulando la lucha contra el sistema y las estructuras institucionales. Así pues, es en gran medida dicha desarticulación de la visión y misión política del feminismo la que permite la emergencia de un conjunto desagregado de individuales frente a la existencia de un sujeto político. De este modo, si bien históricamente el feminismo se ha encargado de poner en evidencia las desigualdades de género y las contradicciones inherentes al neoliberalismo, al capitalismo y al sistema en sí mismo; con el nuevo feminismo neoliberal (Rottenberg, 2013, 2018) nos estamos enfrentando a un nuevo estadio de dicha corriente -que convive con muchas otras- que parece mostrar ciertas simpatías con la lógica neoliberal.

Nos encontramos en una fase donde las contradicciones inherentes a dichos sistemas se superan mediante la autogestión, la superación personal de las barreras y la consecución de la felicidad individual. Con lo que apelando a la dimensión individual se pretende transformar lo colectivo. Consecuentemente, la lucha por la igualdad se desarrolla de un modo totalmente apolítico y conteniendo un discurso 
feminista edulcorado. Se consigue que las mujeres individuales se hagan cargo de problemas que no son individuales, sino colectivos, y que como tales, deberían ser abordados desde las estructuras estatales, económicas o de cualquier otra índole. Los debates en torno a temas como las técnicas de reproducción asistida, los vientres de alquiler, la prostitución, la pornografía, etc. se vuelven centrales en la actualidad, encontrando posturas muy diversas dentro de los feminismos. A continuación, trataremos las diferentes propuestas que realizan las autoras en torno al tema monográfico "Cuerpos en venta", visiones que pretenden ser una puerta abierta a la diversidad de miradas que se dan en torno a estas cuestiones de vital importancia para la lucha feminista actual.

\section{Cuerpos y dominación en el marco neoliberal}

En este apartado trataremos de adentrarnos en la relación existente entre la dominación de los cuerpos de las mujeres y su posición en la sociedad dentro del marco neoliberal, que como hemos dicho, se caracteriza por la precariedad, el riesgo y el individualismo. Para esto, introduciremos los trabajos de diversas autoras que nos proponen, desde disciplinas tan diversas como la filosofía, el derecho, la historia, la teoría del arte y los estudios literarios, una mirada propia sobre las diferentes dimensiones de la dominación sobre los cuerpos de las mujeres en el contexto actual.

Comenzamos con Isabel Balza Múgica, quien abre este monográfico con su reflexión sobre los vínculos entre la dominación de las mujeres y la de los animales no humanos, en su trabajo titulado «Una biopolítica feminista de la carne: la gestación subrogada como ejemplo de los vínculos de opresión entre las mujeres y los animales no humanos». Balza entiende que gran parte de la violencia patriarcal contra mujeres y animales es su conversión en meros objetos. Para reflexionar de forma crítica sobre esta cuestión, la autora propone una distinción biopolítica, cuerpo/carne, frente a las oposiciones zoé/bíos (Agamben, 1995) o persona/no persona (Esposito, 2004). A partir de estas construcciones la autora articula una biopolítica feminista de la carne para analizar la categoría de carne en su aspecto más negativo y cosificador, examinando la gestación subrogada como ejemplo de una biopolítica de la carne que muestra las semejanzas entre la opresión de las mujeres y la de los animales no humanos.

Seguidamente encontramos el trabajo de Lorena García Saiz, quien aborda «La congelación de óvulos en el ámbito laboral por causas sociales: nueva estrategia empresarial para controlar el cuerpo de la mujer». En su artículo, la autora se centra en las empresas que, dentro del territorio del Estado español, promocionan la congelación de los óvulos entre sus trabajadoras, sobre todo entre las directivas o gerentes. Así pues, García Saiz analiza cómo esta operación sirve para que la maternidad no sea una dificultad a la hora de desarrollar la carrera profesional de las mujeres dentro de dichas empresas. Al mismo tiempo, la autora afirma que dicha estrategia contribuye al control de los cuerpos de las mujeres para favorecer los intereses de las organizaciones empresariales, bajo la excusa de ser una herramienta 
que favorece la libertad de sus empleadas a la hora de trazar su itinerario personal y laboral.

Más adelante, nos adentramos en una cuestión de gran interés en el estudio de las relaciones entre cuerpos y mercado, esto es, la pornografía. En este tema encontramos dos reflexiones realizadas desde diferentes campos. En primer lugar, Mónica Alario Gavilán analiza «La influencia del imaginario de la pornografía hegemónica en la construcción del deseo sexual masculino prostituyente: un análisis de la demanda de la prostitución». En su artículo, la autora analiza la construcción del deseo sexual masculino prostituyente como una de las causas centrales de la mercantilización de los cuerpos de las mujeres. Para ello, estudia cómo se construye la masculinidad hegemónica y qué función cumple en ella la sexualidad, analizando en qué medida la pornografía hegemónica contribuye a cosificar a las mujeres, colaborando en la construcción de un deseo sexual masculino que puede llevar a los varones al consumo de prostitución.

En segundo lugar, tenemos el trabajo de Elena Monzón Pertejo titulado «El cuerpo de María Magdalena: representaciones, pornografía y feminismo», donde la autora reflexiona en torno a la relación existente entre pornografía y religión. Desde su perspectiva, ambas esferas han estado íntimamente vinculadas desde hace siglos en el cristianismo occidental. En esta convergencia, sostiene la autora que las representaciones de mujeres bíblicas han jugado un papel esencial y, entre ellas, una de las figuras más destacadas ha sido la de María Magdalena. Por esta razón, en su artículo analiza el uso del cuerpo de María Magdalena como objeto pornográfico, trazando un breve recorrido diacrónico desde el siglo XVI hasta la cultura audiovisual del siglo XXI, centrando la atención en la película Sacred Flesh (Nigel Wingrove, 2000) en donde se da un giro a la utilización de esta figura.

Enmarcando en cierto modo los trabajos expuestos previamente, la reflexión de María Ávila Bravo Villasante pone de manifiesto la estrategia de resignificación lingüística elaborada por el patriarcado neoliberal para invisibilizar todos aquellos significantes que puedan poner en evidencia lo que de facto opera en la prostitución de mujeres y en la gestación subrogada: la reificación de las mujeres. En su artículo «La importancia del lenguaje en el proceso de reificación de las mujeres», la autora se centra en la reacción antifeminista que se produce en Estados Unidos en la década de los ochenta y las inversiones lingüísticas puestas en marcha por la nueva derecha contra el movimiento feminista. Más tarde realiza una reflexión comparativa entre dicho momento histórico y el que operan en el momento actual.

Por otro lado, Mireia Ferrer Álvarez nos introduce en el mundo del arte con su artículo «Cuerpos propios. Antagonismos en el arte de performance femenina en la época del giro performativo». En dicho artículo, Ferrer aborda performances realizadas por mujeres artistas en los últimos 50 años en el ámbito artístico norteamericano, europeo y latinoamericano cuyo significado gira en torno a la construcción patriarcal del cuerpo femenino. A través de dichas performances, la autora pretende hacernos reflexionar sobre la constante agresión y devastación a la que son sometidas las mujeres a través de la conquista y dominación de su cuerpo. 
Abriendo paso a un campo más literario, Gloria Luque Molla nos presenta su investigación titulada "'Sobre mi cadáver". Un análisis sobre el papel del cuerpo en la obra de Mona Hatoum», donde se centra en la obra de dicha artista, quien supuso un punto de inflexión en el desarrollo del arte contemporáneo (Ballester, 2012). Según la autora, Mona Hatoum, artista que ha explorado temas como el género o la propia concepción del hogar desde marcos expresivos muy diversos, utiliza el cuerpo para desafiar al público a atender a problemas acuciantes. Así pues, Luque se centra en el empleo que Hatoum hace del cuerpo como vía de expresión para más tarde atender a las tres dimensiones del cuerpo humano que la artista cuestiona, evidenciando las restricciones sociales y culturales que condicionan nuestro uso del mismo.

La aportación de Lydia de Tienda Palop titulada «Cultura y cuerpo femenino. Aplicación de las categorías orientalistas a la obra Viento del Este, Viento del Oeste de Pearl S. Buck», se centra en el análisis de la novela del mismo título. De Tienda identifica en dicha obra los principios de las tesis expuestas por Edward Said en su obra Orientalismo (2003). Así, la autora sostiene que es posible reconocer estas mismas categorías epistemológicas orientalistas en la configuración de los relatos constitutivos de la esencia femenina. El artículo se centra en tres paralelismos entre las tesis orientalistas y la construcción de la esencia femenina: a) La dicotomía Oriente-Occidente; b) la bipolaridad femenino-masculino; y c) la dialéctica cuerpo-alma, que muestran la perspicaz manera en que la narrativa corporal subyace a la configuración de la autocomprensión existencial femenina.

Vicente Ordoñez Roig reflexiona en torno a «El cuerpo del delirio: Antígona, Zambrano, Femen». En su trabajo, aborda desde una perspectiva filosófica el nexo causal que existe entre el cuerpo entendido como espacio de resistencia política feminista y la asimilación de ese cuerpo a través de un entramado normativo y clínico que acusa de 'locas', 'perturbadas' o 'delirantes' a quienes se posicionan contra los distintos dispositivos del poder. A la hora de trazar dicho nexo, Ordoñez estudia la reacción simbólica de Antígona y el veredicto de locura que recae sobre ella, el intento por reintegrar la locura y el delirio a la vida consciente del ser humano en la obra de María Zambrano, y la lucha cuerpo a cuerpo de Femen (Reverter-Bañón, 2016). Una de las principales conclusiones de esta reflexión es que la locura actúa como un dispositivo disciplinario que se apropia de la corporeidad femenina en su intento por acabar con los estereotipos de género vigentes.

Seguimos con Amalia Rosado Orquín, quien abre paso a la reflexión más histórica con su trabajo «La cosificación de las mujeres como instrumento de una ideología perversa: los cuerpos del fascismo», donde explora algunas de las prácticas que el Estado nazi reservó a las mujeres españolas. La deportación, la prostitución, la esterilización, la experimentación, el trabajo esclavo o el exterminio fueron algunas de las prácticas que estas mujeres sufrieron a manos de la dominación nazi. Rosado explica las consecuencias del fascismo en las mujeres y la importancia de su cuerpo como una herramienta utilizada al servicio del régimen nazi mediante el análisis de los discursos nacionalsocialistas sobre el modelo de género. 
Como se puede observar, las aportaciones que conforman la sección monográfica de «Cuerpos en venta» son de una gran heterogeneidad temática y también diversa en cuestión de aproximaciones. Sin embargo, consideramos que todas comparten una dimensión: incentivar la reflexión sobre la mercantilización de los cuerpos de las mujeres en el escenario actual.

\section{La cuestión de género desde una perspectiva multidisciplinar}

Abriendo la sección de miscelánea encontramos el trabajo de Marina GarcíaGranero, titulado «Injusticias de género en tiempos de neoliberalismo. El planteamiento de Nancy Fraser». A través de la teoría de la justicia propuesta por la filósofa política Nancy Fraser (1989, 1997, 2008, 2015), la autora construye una contundente crítica al neoliberalismo desde una perspectiva de género. Poniendo énfasis en las categorías de la redistribución, el reconocimiento y la representación, Fraser pretende elucidar cómo reconectar la teoría y la práctica feministas en una época de acuciado neoliberalismo. En este sentido, García-Granero pone en valor la teoría de la justicia de dicha autora a la hora de elaborar una crítica a un supuesto feminismo neoliberal -mencionado anteriormente- el cual deja intactas las estructuras de dominación económicas y promueve únicamente el empoderamiento de una minoría privilegiada de mujeres. Sin duda, se trata de una reflexión filosófica de gran relevancia en unos tiempos de aguda mercantilización de la vida.

Seguidamente, contamos con el trabajo de María Ángeles Goicoechea Gaona y Victoria Goicoechea Gaona titulado «Diversidad afectiva: visibilizar la homosexualidad femenina». En su investigación ambas autoras abordan los discursos sociales en referencia a las mujeres lesbianas en el contexto de Colima (México). A través de un trabajo de investigación sociológica cualitativa, las autoras consiguen poner en evidencia la necesidad de visibilizar la diversidad afectiva y sexual, en general, y la realidad de las mujeres lesbianas, en particular, doblemente discriminadas por ser mujeres y por su deseo en discrepancia con el sistema heteronormativo. Así pues, el fin último de su trabajo de investigación es sensibilizar a la ciudadanía con el propósito de que pueda comprender la realidad de aquellas personas que no coinciden con la herteronormatividad.

Más adelante, Patricia Oliva Barboza aborda desde el mundo del arte «Las llamadas "Mujeres públicas". Historizando a través del arte». En su artículo analiza la obra «Vacío», creada y dirigida por Roxana Ávila (actriz y dramaturga costarricense), desde una perspectiva feminista y crítica. Cabe señalar que este trabajo complementa otros análisis previos en la misma línea (Oliva, 2015). A través de dicho análisis la autora trata de reconstruir críticamente la expropiación de los cuerpos de las mujeres, enfatizando en un extracto de la historia de la prostitución en Costa Rica. Así pues, Oliva se centra en el proceso discursivo, médico y estatal mediante el cual las mujeres fueron etiquetadas de «mujeres públicas». Así pues, Oliva sostiene que existe una estrecha relación entre la construcción de la locura femenina, la patologización y la intención socio-política y religiosa de etiquetar a las mujeres entre «públicas», es decir insanas, y las mujeres «madres y esposas». 
Victoria Puchal Terol nos presenta su propuesta de investigación «Priscilla Horton, la ninfa de cabellos dorados: una actriz convertida en gerente teatral en el Londres victoriano». En este artículo la autora aborda la vida de Priscilla Horton (1818-1895), actriz y gerente de teatro en el Londres del siglo XIX. A través de un trabajo de puesta en valor de la vida de Horton, la autora nos permite descubrir la situación social de las actrices de la época y las dificultades a las que debían hacer frente. Además, Horton impulsó un nuevo género a mediados del siglo XIX, el «entretenimiento de salón» o drawing-room entertainment, atrayendo incluso a la élite que consideraba el teatro como vulgar e inmoral.

Por otro lado, Yasmina Romero Morales se centra en estudiar los «Poderes de perversión: la femme fatale colonial de la narrativa española del siglo XX». En su artículo, la autora trata de analizar uno de los arquetipos clave de la narrativa colonial española: la femme fatale marroquí. Teniendo en mente que es imposible representar a Oriente sin prejuicios, tal y como sostuvo el propio Edward W. Said $(2003,2012)$, Romero trata de colaborar en la representación más fiel del objeto, mediante el análisis de la construcción de la femme fatale marroquí desde los principios rectores del análisis desde la perspectiva de género, los estudios culturales y las teorías postcoloniales.

Más adelante Thelma Elena Pérez Álvarez nos propone su trabajo titulado «¿Diversidad, empoderamiento y libre elección? Una mirada a representaciones de las mujeres en el spot Bonafont, fluye en ti (México, 2016)». En este artículo se estudian los modos en que fueron encarnados los personajes femeninos del spot Bonafont, fluye en ti de la marca de agua embotellada de Grupo Danone. Mediante un análisis se identifican las contradicciones discursivas de un relato audiovisual que celebra la diversidad de formas de ser mujer, el empoderamiento y la libre elección de las mujeres. Con ello se desvela cómo este texto audiovisual abona a la construcción y difusión de productos culturales que pueden asociarse con expresiones de violencia simbólica contra las mujeres en México.

Por otro lado, Victoria Aragón García cierra la sección de miscelánea con su artículo "Construir justicia social: enfoque del agua con perspectiva de género», donde se analiza la relación entre agua y género, destacando la vulneravilidad de las mujeres y las razones de su exclusión en los órganos de decisión. En este sentido y tomando como marco de referencia el agua y su problemática social y ambiental, la autora señala la importancia de implementar el enfoque de género en las políticas públicas de cara a una mayor sostenibilidad y bienestar social.

En la sección «Retrat», Teresa Sorolla Romero nos introduce en la obra de Jane Campion, afamada directora y guionista de cine ampliamente conocida por sus obras The Piano (1993),

The Portrait of a Lady (1996), In the Cut (2003) o Top of the Lake (2013-2017), entre muchas otras. En este retrato, Sorolla realiza una aproximación a los principales rasgos de la obra cinematográfica de Campion, poniendo el acento en su forma de representar las experiencias de las mujeres y sus cuerpos en diferentes contextos históricos, siempre haciendo alarde de una gran sensibilidad con respecto a los temas que afectan a dicho grupo. 
En la sección de «Creació literària» encontramos una reflexión realizada por Silvia Martínez Cano y Pepa Santamaría, en representación de la Asociación Internacional de Mujeres Artistas EmPoderArte. El texto se centra en una exposición de arte organizada por dicha asociación en torno al debate social sobre la maternidad subrogada y su repercusión en el cuerpo de las mujeres. A través de este proyecto colectivo de denuncia y reflexión, EmPoderArte trata de realizar un trabajo de concienciación y visibilización de este tema en los ámbitos culturales y sociales, plateando dilemas tanto éticos como jurídicos, que subrayan la posición de vulnerabilidad de las mujeres en estas transacciones comerciales.

Por último, en la sección «Llibres», contamos con cuatro recensiones de obras de gran actualidad en el campo de los estudios feministas y de género. En primer lugar, Eduardo Torres Alonso reseña la obra El silencio habla. Democracia, paridad y género de Lucero Saldaña (2015). En segundo lugar, Javier Cuevas del Barrio analiza la obra de Sirin Adlbi Sibai titulada La cárcel del feminismo. Hacia un pensamiento islámico decolonial (2017). En tercer lugar, Marc Salvador Queral realiza una recensión de la reedición de la conocida obra Feminism is for everybody: passionate politics (2017), de la autora bell hooks. En cuarto y último lugar, Josefa García Sánchez analiza Querida Ijeawele. Cómo educar en el feminismo (2017) de la afamada autora Chimamanda Ngozi Adichie.

\section{Conclusión}

Alolargo de esteartículohemos tratado de reflexionar en torno a la mercantilización de los cuerpos femeninos que hoy parece estar tan a la orden del día, demostrando que el cuerpo es uno de los temas de reflexión más importantes de la teoría feminista en los siglos XX y XXI. Al mismo tiempo, hemos tratado de enmarcar y comprender el marco de sentido en el que se produce dicha mercantilización de los cuerpos. Para esto hemos incidido en dos de las cuestiones que otorgan entidad propia a la época neoliberal, esto es, la precariedad y el individualismo que promueven una lógica de la elección individual completamente mercantilista y despolitizada.

En su intersección con la cuestión de género, dicha situación dibuja un escenario donde parte del feminismo se ve influenciado por la lógica neoliberal. Hoy, esta realidad nos invita a reflexionar sobre ciertas cuestiones de gran relevancia social como por ejemplo las técnicas de reproducción asistida (Martí Gual, 2011, 2014), los vientres de alquiler (Nuño, 2016; Guerra Palmero, 2017), la prostitución (Nuño, De Miguel y Fernández, 2017), etc.

Sin embargo, lejos de ofrecer una mirada cerrada sobre estas cuestiones, consideramos que es necesario seguir planteándonos dichos interrogantes desde una mirada que, desde la perspectiva crítica de género (Medina-Vicent y Reverter-Bañón, 2016), pretenda ser transformadora y que camine a la justicia social. Esperamos que este monográfico sea una valiosa contribución para seguir transitando dicho camino. 


\section{REFERENCIAS BIBLIOGRÁFICAS}

Adlbi, Sirin (2017). La cárcel del feminismo. Hacia un pensamiento islámico decolonial, México D.F.: Akal.

AgAmben, Giorgio (1995). Homo Sacer. El poder soberano y la nuda vida, València: PreTextos.

Alonso, Luis Enrique y Carlos Jesús Fernández Rodríguez (2013). Los discursos del presente. Un análisis de los imaginarios sociales contemporáneos, Madrid: Siglo XXI.

Alonso, Luis Enrique y Carlos Jesús Fernández Rodríguez (2018). Poder y sacrificio. Los nuevos discursos de la empresa, Madrid: Siglo XXI.

BALlESTER, Irene (2012). El cuerpo abierto. Representaciones extremas de la mujer en el arte contemporáneo, Gijón: Ediciones Trea.

BALzA, Isabel (2014). «Los feminismos de Spinoza: corporalidad y renaturalización» en Daimon. Revista Internacional de Filosofía, N ${ }^{\circ}$ 63, pp. 13-26.

Berardi, Franco (2003). La fábrica de la infelicidad, Madrid: Traficantes de sueños.

Boltanski, Luc y Ève Chiapello. (2002). El nuevo espíritu del capitalismo. Madrid: Akal.

CARBAjo, Diego (2014). Vivir en la precariedad. Trayectorias y estrategias residenciales de la juventdu en la Comunidad Autçonoma del País Vasco, Bizkaia: Universidad del País Vasco.

De Miguel Álvarez, Ana (2015). Neoliberalismo sexual. El mito de la libre elección, Madrid: Cátedra.

Esposito, Roberto (2004). Bíos. Biopolítica y filosofía, Buenos Aires: Amorrortu.

Ferguson, Michaele L. (2010). «Choice Feminism and the Fear of Politics» en Perspectives on Politics Vol.8, No1, pp. 247-253. doi:10.1017/S1537592709992830.

Fraser, Nancy (1989). Unruly Practices: Power, Discourse and Gender in Contemporary Social Theory, Minneapolis: University of Minnesota Press.

FrASER, Nancy (1997). Iustitia Interrupta. Reflexiones críticas desde la posición «postsocialista», Bogotá: Siglo del Hombre Editores.

FrAser, Nancy (2008). Escalas de justicia, Barcelona: Herder.

FrASER, Nancy (2015). Fortunas del feminismo. Del capitalismo gestionado por el Estado a la crisis neoliberal, Madrid: Traficantes de Sueños.

GARcía FERrer, Borja (2017). «El precio del progreso: de la «virtualización del mundo» al «zombismo hiperindividualista»» en Recerca. Revista de pensament $i$ anàlisi, 20, pp. 105-126. doi: 10.6035/Recerca.2017.20.6

Gill, Rosalind (2007). «Postfeminist Media Culture Elements of a Sensibility» en European Journal of Cultural Studies, Vol.10, N22, pp. 147-166. doi: $10.1177 / 1367549407075898$.

Guerra Palmero, María José (2017): «Contra la mercantilización de los cuerpos de las mujeres. La "gestación subrogada" como nuevo negocio transnacional» en Dilemata. Revista Internacional de Éticas Aplicadas, No26, pp. 39-51.

Hirshman, Linda R. (2006). Get to Work: A Manifesto for Women of the World.New York: Viking.

Ноокs, bell (2017). Feminism is for everybody: passionate politics, Madrid: Traficantes de sueños.

LAval, Christian y Pierre DARdot (2013). La nueva razón del mundo. Ensayo sobre la sociedad neoliberal, Barcelona: Gedisa. 
Martí GuAl, Ana (2011). Maternidad y técnicas de reproducción asistida: un análisis desde la perspectiva de género, de los conflictos y experiencias de las mujeres usuarias. Castelló de la Plana: Universitat Jaume I.

MARTí GuAL, Ana (2014). «Entre la tradición, la gestión del tiempo y la innovación: discursos sobre la maternidad de las mujeres usuarias de reproducción asistida» en Pérez Sedeño, Eulalia y Esther Ortega Arjonilla (2014). Cartografías del cuerpo : biopolíticas de la ciencia y la tecnología, Madrid: Ediciones Cátedra, pp. 245-308.

McRoвbie, Angela (2004). «Post-Feminism and Popular Culture» en Feminist Media Studies, Vol.4, N³, pp. 255-264. doi:10.1080/1468077042000309937.

McRoвbie, Angela (2009). The Aftermath of Feminism: Gender, Cultural and Social Change, London: Sage.

MedinA-Vicent, Maria (2018). «Flirting with Neoliberalism: The Transfiguration of Feminist Political Awareness» en NORA. Nordic Journal of Feminist and Gender Research, Vol. 25, No1, pp. 69-75. doi: 10.1080/08038740.2018.1424728

MedinA-Vicent, Maria y Sonia Reverter-Bañón (2016). «La perspectiva de género como una mirada crítica al mundo» en Asparkía. Investigació Feminista, N 29 , pp. 11-16. doi: 10.6035/Asparkia.2016.29.1

MuÑoz-Rodríguez, David y Antonio SAntos-Ortega (2017). «Las cárceles del capital humano: trabajo y vidas precarias en la juventud universitaria» en Recerca. Revista de pensament $i$ anàlisi, No20, 59-78. doi: 10.6035/Recerca.2017.20.4

Muñoz-Rodríguez, David y Antonio Santos-Ortega (2018). En las cárceles del capital humano. Nuevas precariedades y formas de subjetivación de los procesos contemporáneos de precarización, València/Málaga: Baladre/Zambra.

Ngozi Adichie, Chimamanda (2017). Querida Ijeawele. Cómo educar en el feminismo, Barcelona: RandomHouse.

NuÑo, Laura (2016). «Una nueva cláusula del Contrato Sexual: vientres de alquiler» en Isegoría, N55, pp. 683-700.

Nuño, Laura y Ana De Miguel Álvarez (dirs.) y Lidia Fernández (coord.) (2017). Elementos para una teoría crítica del sistema prostitucional, Granada: Editorial Comares.

Oliva, Patricia (2015). «Desde lo profundo de sus obras: Un análisis feminista sobre la expropiación/patologización del cuerpo de las mujeres» en Rupturas, Vol. 7, N2, pp. 163-191.

REVERTER-BAÑón, Sonia (2001): «Feminismo y democracia: una crítica antifundamentalista» en Recerca. Revista de pensament i anàlisi, Nº1, pp. 95-108.

Reverter-Bañón, Sonia (2016). «El feminismo dislocado: el caso FEMEN», en Eikasía, N $\mathrm{N}^{\mathrm{0}} 70$, pp. 159-187.

REVERTER-BAÑón, Sonia (2017). «Cosmopolitismo Feminista contra Globalización» en Araucaria. Revista Iberoamericana de Filosofía, Política y Humanidades, N¹9, pp. 301-25. https:/ / doi.org/10.12795/araucaria.2017.i37.15

Rottenberg, Catherine (2013). «Neoliberal Feminism and the Future of Human Capital» en Signs: Journal of Women in Culture and Society, N², Vol. 2, pp. 32948. doi: $10.1086 / 688182$

RotTenberG, Catherine (2014). «Happiness and the Liberal Imagination: How Superwoman Became Balanced» en Feminist Studies, Vol.40, Nº1, pp. 144-68. 
RotTenberg, Catherine (2018). The Rise of Neoliberal Feminism, Oxford: Oxford University Press.

SAID, Edward W. (2003). Orientalismo, Barcelona: Debolsillo.

SAID, Edward W. (2012). Cultura e imperialismo, Barcelona: Anagrama.

SAldaña, Lucero (2015). El silencio habla. Democracia, paridad y género, México D.F.: Miguel Ángel Porrúa-Senado de la República.

SwirsKy, Jill M., y D. J. Angelone (2016). «Equality, Empowerment, and Choice: What does Feminism Mean to Contemporary Women?» en Journal of Gender Studies, Vol.25, N4, pp. 445-460. doi:10.1080/09589236.2015.1008429.

ThwaItes, Rachel (2017). «Making a Choice or Taking a Stand? Choice Feminism, Political Engagement and The Contemporary Feminist Movement» en Feminist Theory, Vol. 18, No1, pp. 55-68. doi: 10.1177/1464700116683657. 